\title{
Misinformation about Covid-19 Vaccines on Social Media: Rapid Review
}

\author{
Ingjerd Skafle, ${ }^{1 *}$ Anders Nordahl-Hansen, ${ }^{2}$ Daniel S. Quintana, ${ }^{3}$ \\ Rolf Wynn, ${ }^{4}$ and Elia Gabarron ${ }^{2}$
}

\begin{abstract}
The development of COVID-19 vaccines has been crucial in fighting the pandemic. Misinformation about COVID-19 vaccines on social media is a major challenge, as this is thought to contribute to vaccine hesitancy. Here, we review the research on misinformation about COVID-19 vaccines spread on social media platforms. The review is registered with the PROSPERO international register of systematic reviews (CRD42021277524). We performed a literature search on 9 September 2021 and searched PubMed, PsycINFO, ERIC, Embase, Cochrane Library, and the Cochrane COVID-19 Study Register. We included publications in peer-reviewed journals that fulfilled all following criteria: Original empirical studies, studies that assessed social media and misinformation, studies that dealt with vaccine hesitancy, and studies about the COVID- 19 vaccine. Publications were excluded if data were gathered before Phase III in the first COVID-I9 vaccine development (from July 272020 to November 13, 2020). The narrative qualitative synthesis was undertaken with the guidance of the PRISMA 2020 Statement and the Synthesis Without Meta-analysis reporting guideline. The risk of bias was assessed according to The Joanna Briggs Institute (JBI) Critical Appraisal tool. Ratings of the certainty of evidence were based on recommendations from the GRADE Working Group. The search amounted to 757 records, with 45 articles selected for the review. There are many types of misinformation that are spread on social media platforms. We identified three main areas of misinformation: Medical misinformation, vaccine development, and conspiracies. To prevent these misconceptions from taking hold, health authorities should openly address and discuss these false claims with both cultural and religious awareness in mind. Our review showed that there is a need to examine the effect of social media misinformation on vaccine hesitancy with a more robust experimental design. Furthermore, our review also demonstrated that more studies are needed from the Global South and on other social media platforms than the major platforms such as Twitter.
\end{abstract}

\section{Keywords}

Social Media, Misinformation, COVID-19 Vaccines, Vaccination Hesitancy, Autism Spectrum Disorder

\footnotetext{
I Faculty of Health, Welfare and Organisation, Østfold University College, Norway

${ }^{2}$ Department of Education, ICT and Learning, Østfold University College, Norway

${ }^{3}$ Department of Psychology, University of Oslo, Norway; NORMENT Centre for Psychosis Research and KG Jebsen Centre for Neurodevelopmental Disorders, University of Oslo, Norway; NevSom, Department of Rare Disorders, Oslo University Hospital, Norway

${ }^{4}$ Department of Clinical Medicine, The Artic University of Norway
}

\section{*Corresponding Author:} Ingjerd Skafle. Faculty of Health, Welfare, and Organisation. Østfold University College, B R A Veien 4, I757 Halden, Norway. Email: Ingjerd.skafle@hiof.no 


\section{Introduction}

An unprecedented global effort has been undertaken to develop vaccines at an extraordinary speed. However, there is a grave concern that vaccine hesitancy will be a major obstacle to reaching herd immunity. In 2019, the WHO had already named vaccine hesitancy as one of ten threats to global health. ${ }^{1}$ Global vaccine-distribution equity is a major challenge. Figures from December 2021 show that 54.8\% of the world's population has received at least one dose of a COVID-19 vaccine. ${ }^{2}$ However, only $6 \cdot 2 \%$ of people in the Global South have received at least one dose. ${ }^{2}$ The rate of people receiving the COVID-19 vaccine in some high-income countries where vaccines are available and free has dropped. ${ }^{3}$ The World Health Organization (WHO) reiterates that vaccines remain critical and are considered effective against severe disease and death. ${ }^{4}$

The reasons behind COVID-19 vaccine hesitancy are complex. Fear of side effects and concerns about the pace at which the vaccines were developed have been cited as primary reasons behind the hesitancy. ${ }^{5}$ In addition, misinformation about COVID-19 and vaccines is spread on social media platforms at a rate that has made the WHO coin the phrase "infodemic". ${ }^{6}$ An Infodemic is "too much information including false or misleading information in digital and physical environments during a disease outbreak." This infodemic plays a crucial role in public health when it comes to understanding the role of social media and COVID-19. ${ }^{8}$ A well-known false claim is that the Measles, Mumps, and Rubella vaccine (MMR) can cause autism. ${ }^{9}$ The claim has since been scientifically refuted many times but is still stated as a major concern for some parents. ${ }^{10}$ Motta and Steccula ${ }^{11}$ examined American public opinion data on MMR safety collected before and after a retracted 1998 study that linked autism to MMR. The researchers detected a statistically significant rise in public concern about MMR safety following the retracted study and the media attention it received. This suggests that misleading vaccine information can impact public confidence in vaccines, and can cause skepticism about vaccines in general. Therefore, we also examined whether misinformation on social media now linked autism to the COVID-19 vaccines. Furthermore, vaccine hesitancy based on misinformation seems to be a worldwide phenomenon regardless of the uneven distribution of COVID-19 vaccines. ${ }^{4}$ Social media plays a crucial role in disseminating both information and misinformation about infectious diseases and vaccines $^{12}$ Wilson and Wiysone showed in a global cross-national analysis of geographically-coded tweets and vaccination rates from 166 countries, that there was a significant relationship between social media 
use and vaccine hesitancy. ${ }^{13}$ There has, however, been a joint effort by several of the largest social media platforms and technology companies to combat the spread of misinformation about COVID-19. ${ }^{14}$ In this study, we will review studies on social media misinformation on COVID-19 vaccines. The following questions guide our inquiry: What is known about misinformation regarding COVID-19 vaccines spread on social media platforms? What is known about the effects of misinformation about COVID-19 vaccines spread on social media platforms? What is known about social media misinformation on COVID-19 vaccines concerning autism spectrum disorder (ASD)?

\section{Methods}

\section{Design}

We followed the guidance from Cochrane Rapid Reviews. ${ }^{15}$ We chose a rapid review protocol in line with the recommendations by Cochrane, that is, the need "for timely evidence for decision-making purposes including to address urgent and emergent health issues and questions deemed to be of high priority". ${ }^{15}$ The need to address vaccine hesitancy towards COVID-19 vaccines is an emergent health issue. The narrative qualitative synthesis was undertaken with the guidance of the PRISMA 2020 Statement ${ }^{16}$ and the Synthesis Without Meta-analysis (SWiM) reporting guideline. ${ }^{17}$

\section{Search Strategy and Selection Criteria}

We used the following search string in the rapid review:

“misinformation” OR “disinformation” OR “information” AND “social media” OR "Facebook” OR

“Twitter” OR “Instagram” OR "WhatsApp” OR “Telegram” OR “Tumblr” OR "Pinterest” OR "YouTube" OR "VKontakte" OR "Snapchat" OR "TikTok" OR "Weibo" OR "WeChat" OR "Reddit" AND "covid* OR "corona* OR "pandemic" OR "Sars-CoV-2" AND "vaccine*” OR "vaccination*”. No date or language limitations were used. The full search strategy is available in supplementary appendix.

We included publications in peer-reviewed journals that fulfilled all following criteria: Original empirical studies, studies that assessed social media and misinformation, studies that dealt with vaccine hesitancy, studies about the COVID-19 vaccine. There were no language restrictions set. Publications were excluded if data were gathered before Phase III in the first COVID-19 vaccine development (from July 
272020 to November 13, 2020). ${ }^{18}$ The reason behind this particular criterion was that we wanted to include studies from the point when information about the forthcoming vaccines was reaching the public.

\section{Data Collection Process and Extraction}

The review is registered in PROSPERO (CRD42021277524). Systematic searches in the databases PubMed, PsycINFO, ERIC, Embase, Cochrane Library, Cochrane COVID-19 Study Register were conducted by an information retrieval specialist on 9 September 2021. Duplicates were identified and removed. We used Rayyan as the chosen screening tool. Titles and abstracts were screened by two reviewers. Conflicting assessments were solved through discussions. 120 titles qualified for full-text review. Two reviewers did a further assessment of the eligibility of these 120 full-text records and conducted a pilot exercise using the same 10 full-text articles to calibrate and test the review form. Subsequently, the two reviewers read 55 articles each. After the screening, both assessed the articles the other had excluded. Another reviewer assisted with conflicts and discussed doubts surrounding included or excluded articles. 45 articles were included for data extraction. The data extraction from the identified articles involved two reviewers, where one extracted data using a piloted form and the other checked for the correctness and completeness of extracted data. Data from the included articles were extracted with regards to design and study population, type of misinformation, the effect of misinformation, misinformation about autism, ethical considerations, and social media channel. The agreed evidence was then synthesized narratively.

To synthesize the gathered knowledge about types of misinformation, a thematic analysis was performed. ${ }^{19}$ The types of misinformation were analysed and coded and then categorized into main themes by one reviewer. The codes and themes were discussed and approved by two additional reviewers. The planned group of studies that involve autism was removed since only one study contained this information.

\section{Assessment of Risk of Bias}

The risk of bias was graded according to The Joanna Briggs Institute (JBI) Critical Appraisal tool "Checklist for Analytical Cross-sectional Studies" 20 by one experienced reviewer. The evaluation was 
based on answers to eight questions. The studies were classified to have either low (score 7-8), moderate (score 4-6), or high (score 1-3) risk of bias.

\section{Assessment of the Quality of the Evidence}

One experienced reviewer assigned certainty of evidence ratings based on recommendations by the GRADE Working Group. ${ }^{21}$ The included studies that looked at associations were given a narrative GRADE score related to the outcome "Association between social media misinformation and vaccine hesitancy". The level of quality of evidence was classified as very low, low, moderate, or high.

\section{Data Synthesis}

The narrative synthesis was undertaken with the guidance of the PRISMA 2020 Statement ${ }^{16}$ and the Synthesis Without Meta-analysis reporting guideline. ${ }^{17}$ In the synthesis, findings from our included studies were grouped according to study design, population, and social media sample, types of social media, reported types of misinformation, misinformation about autism, the reported effect of the misinformation on vaccine hesitancy, and the assessments of risk of bias and quality of evidence. When synthesizing the findings narratively, studies that have a low risk of bias or high quality of evidence will be highlighted on several occasions.

\section{Role of the Funding Source}

There was no specific funding source for this study.

\section{Results}

We identified 45 relevant studies (Figure 1). The list of excluded articles during the full-text review and the reasons for exclusion are reported in supplementary appendix. The risk of bias in 16 included studies was classified as low according to the JBI critical appraisal tool. ${ }^{22-37}$ Five studies showed a moderate risk of bias. ${ }^{38-41,44}$ Finally, 24 included studies showed a high risk of bias. ${ }^{42,43,45-66}$ 


\section{Identification of studies via databases and registers}

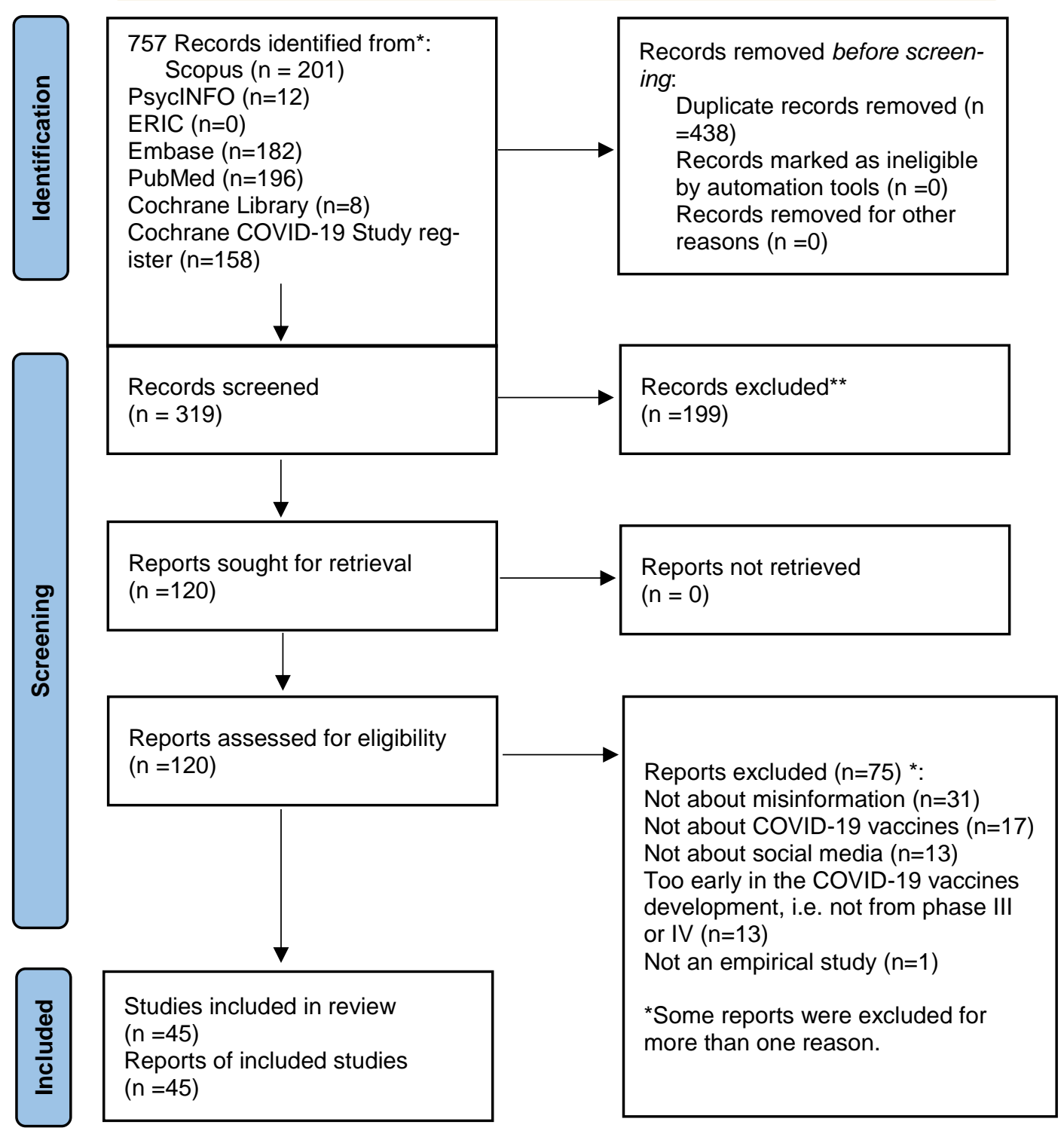

Figure 1: PRISMA 2020 flow diagram for new systematic reviews which included searches of databases and registers only 
Table 1: Studies where data was collected by surveys, observations, and/or interviews ( $\mathrm{n}=\mathbf{2 2}$ )

\begin{tabular}{|c|c|c|c|c|c|c|c|}
\hline Author (year) & Country & Study Period & Study design & $\begin{array}{l}\text { Type of } \\
\text { Social } \\
\text { Media } \\
\end{array}$ & \begin{tabular}{|c|c|}
$\begin{array}{c}\text { Social Media } \\
\text { or population } \\
\text { sample }\end{array}$ \\
\end{tabular} & \begin{tabular}{|c|} 
Type of misinformation re- \\
ported
\end{tabular} & Risk of bias (JBI) \\
\hline $\begin{array}{l}\text { Alibrahim \& } \\
\text { Awad }(2021)^{22}\end{array}$ & Kuwait & $\begin{array}{l}26 \text { March to } \\
26 \text { April } 2021\end{array}$ & $\begin{array}{l}\text { Cross-sectional } \\
\text { study }\end{array}$ & $\begin{array}{l}\text { Not speci- } \\
\text { fied }\end{array}$ & $\begin{array}{l}4147 \text { adults, } \\
18 \text { years or } \\
\text { older }\end{array}$ & $\begin{array}{l}\text { COVID-19 is not a serious } \\
\text { infection that requires vac- } \\
\text { cination }\end{array}$ & Low \\
\hline $\begin{array}{l}\text { Allington et al } \\
(2021)^{23}\end{array}$ & $\begin{array}{l}\text { United } \\
\text { Kingdom }\end{array}$ & $\begin{array}{c}21 \text { November } \\
\text { to } 21 \text { Decem- } \\
\text { ber } 2020\end{array}$ & $\begin{array}{l}\text { Cross-sectional } \\
\text { study }\end{array}$ & $\begin{array}{c}\text { Not speci- } \\
\text { fied }\end{array}$ & $\begin{array}{c}4343 \text { UK resi- } \\
\text { dents, aged } \\
18-75 \text { years }\end{array}$ & $\begin{array}{l}\text { Conspiracy theories (not } \\
\text { specified) }\end{array}$ & Low \\
\hline $\begin{array}{l}\text { Aloweidi et al } \\
\quad(2021)^{24}\end{array}$ & Jordan & \begin{tabular}{|c|}
22 January \\
$202 I$ to 28 \\
February 2021
\end{tabular} & $\begin{array}{l}\text { Cross-sectional } \\
\text { study }\end{array}$ & $\begin{array}{l}\text { Not speci- } \\
\text { fied }\end{array}$ & 646 adults & \begin{tabular}{|c|} 
The vaccines are unsafe; ef- \\
fect of the vaccines on a ge- \\
netic level; causes chronic \\
illnesses; may lead to infer- \\
tility; can affect their off- \\
spring; contains toxic heavy \\
metals and neurotoxic mate- \\
rials; it is a part of a secret \\
research
\end{tabular} & Low \\
\hline $\begin{array}{l}\text { Bhagianadh \& } \\
\text { Arora }(2021)^{25}\end{array}$ & USA & $\begin{array}{l}\text { October-No- } \\
\text { vember } 2020\end{array}$ & $\begin{array}{l}\text { Longitudinal sur- } \\
\text { vey }\end{array}$ & $\begin{array}{c}\text { Not speci- } \\
\text { fied }\end{array}$ & $\begin{array}{l}5784 \text { Medi- } \\
\text { care enrolees, } \\
65 \text { years or } \\
\text { older }\end{array}$ & $\begin{array}{l}\text { Distrust of government nar- } \\
\text { rative about vaccines; vac- } \\
\text { cine will cause COVID-19. }\end{array}$ & Low \\
\hline $\begin{array}{l}\text { Brodiziak et al } \\
\quad(2021)^{26}\end{array}$ & Poland & $\begin{array}{c}\text { January } 26, \text { to } \\
\text { February } 28 \\
2021\end{array}$ & Survey & $\begin{array}{c}\text { Not speci- } \\
\text { fied }\end{array}$ & $\begin{array}{c}635 \text { adult can- } \\
\text { cer patients }\end{array}$ & $\begin{array}{c}\text { The vaccine contains bodies } \\
\text { of aborted children; COVID- } \\
19 \text { does not exist }\end{array}$ & Low \\
\hline $\begin{array}{l}\text { Chadwick et al } \\
\qquad(2021)^{27}\end{array}$ & $\begin{array}{l}\text { United } \\
\text { Kingdom }\end{array}$ & $\begin{array}{c}24 \text { September } \\
\text { to } 17 \text { October } \\
2020 .\end{array} \mid$ & $\begin{array}{l}\text { Cross-sectional } \\
\text { study }\end{array}$ & $\begin{array}{l}\text { Not speci- } \\
\text { fied }\end{array}$ & $\begin{array}{l}5114 \mathrm{UK} \\
\text { adults }\end{array}$ & $\begin{array}{l}\text { Conspiracies } \\
\text { (not specified) }\end{array}$ & Low \\
\hline $\begin{array}{l}\text { Ebrahimi et al } \\
\quad(2021)^{28}\end{array}$ & Norway & $\begin{array}{c}\text { January } 23 \text { to } \\
\text { February } 2 \\
2021 .\end{array}$ & $\begin{array}{l}\text { Cross-sectional } \\
\text { study }\end{array}$ & $\begin{array}{c}\text { Not speci- } \\
\text { fied }\end{array}$ & $\begin{array}{l}4571 \text { Norwe- } \\
\text { gian adults }\end{array}$ & Not specified & Low \\
\hline $\begin{array}{l}\text { Kanyike et al } \\
(2021)^{29}\end{array}$ & Uganda & $\begin{array}{l}\text { Monday } 15 \\
\text { March and } \\
\text { Sunday } 21 \\
\text { March } 2021\end{array}$ & $\begin{array}{l}\text { Cross-sectional } \\
\text { study }\end{array}$ & $\begin{array}{c}\text { Not speci- } \\
\text { fied }\end{array}$ & $\begin{array}{c}600 \text { medical } \\
\text { students, } 18 \\
\text { years or older }\end{array}$ & $\begin{array}{c}\text { Negative information about } \\
\text { Covid-19 }\end{array}$ & Low \\
\hline $\begin{array}{c}\text { Karabela et al } \\
(2021)^{30}\end{array}$ & Turkey & $\begin{array}{c}\text { February } 01 \\
2021, \text { to Feb- } \\
\text { ruary } 28 \\
2021\end{array}$ & $\begin{array}{l}\text { cross-sectional } \\
\text { study }\end{array}$ & $\begin{array}{c}\text { Social } \\
\text { Media } \\
\\
\text { What's } \\
\text { App } \\
\text { YouTube }\end{array}$ & 1216 adults & $\begin{array}{l}\text { Conspiracy theories } \\
\text { (not specified) }\end{array}$ & Low \\
\hline $\begin{array}{l}\text { Park et al } \\
(2021)^{31}\end{array}$ & USA & $\begin{array}{c}\text { Between Oc- } \\
\text { tober and No- } \\
\text { vember } 2020\end{array}$ & $\begin{array}{l}\text { Cross-sectional } \\
\text { study }\end{array}$ & $\begin{array}{c}\text { Not speci- } \\
\text { fied }\end{array}$ & $\begin{array}{l}6478 \text { Medi- } \\
\text { care benefi- } \\
\quad \text { ciaries }\end{array}$ & $\begin{array}{c}\text { The belief that COVID-19 is } \\
\text { not that dangerous }\end{array}$ & Low \\
\hline $\begin{array}{l}\text { Petravic et al } \\
(2021)^{32}\end{array}$ & Slovenia & $\begin{array}{c}\text { 17-27 Decem- } \\
\text { ber } 2020\end{array}$ & $\begin{array}{l}\text { Cross-sectional } \\
\text { study }\end{array}$ & $\begin{array}{l}\text { Not speci- } \\
\text { fied }\end{array}$ & $\begin{array}{c}12,042 \text { Slove- } \\
\text { nian residents, } \\
15 \text { years or } \\
\text { older. } \\
\text { Analysis of re- } \\
\text { sponses from } \\
\text { the } 2320 \text { re- } \\
\text { spondents }\end{array}$ & $\begin{array}{c}\text { The vaccines will cause a } \\
\text { genocide; COVID-19 is the } \\
\text { same as influenza }\end{array}$ & Low \\
\hline
\end{tabular}




\begin{tabular}{|c|c|c|c|c|c|c|c|}
\hline & & & & & \begin{tabular}{|c|}
$(12 \%)$ who \\
answered the \\
open-ended \\
question.
\end{tabular} & & \\
\hline $\begin{array}{l}\text { Sallam et al } \\
(2021)^{33}\end{array}$ & Jordan & $\begin{array}{c}19 \text { January } \\
2021 \text { to } 23 \\
\text { January } 2021\end{array}$ & $\begin{array}{l}\text { Cross-sectional } \\
\text { study }\end{array}$ & $\begin{array}{l}\text { Not speci- } \\
\text { fied }\end{array}$ & $\begin{array}{l}1106 \text { univer- } \\
\text { sity students }\end{array}$ & $\begin{array}{c}\text { Covid-19 was man-made to } \\
\text { enforce vaccinations; Covid- } \\
19 \text { vaccinations intends to } \\
\text { implant microchips into peo- } \\
\text { ple to control them; COVID- } \\
19 \text { vaccination will lead to } \\
\text { infertility; }\end{array}$ & Low \\
\hline $\begin{array}{l}\text { Sallam et al } \\
(2021)^{34}\end{array}$ & $\begin{array}{c}\text { Jordan, } \\
\text { Kuwait, } \\
\text { Saudi } \\
\text { Arabia }\end{array}$ & $\begin{array}{c}4 \text { December } \\
2020 \text { to } 18 \\
\text { December } \\
2020\end{array}$ & $\begin{array}{l}\text { Cross-sectional } \\
\text { study }\end{array}$ & $\begin{array}{c}\text { Facebook, } \\
\text { Instagram, } \\
\text { Twitter, } \\
\text { WhatsApp }\end{array}$ & $\begin{array}{c}3414 \text { respond- } \\
\text { ents }\end{array}$ & $\begin{array}{l}\text { An artificial origin of the vi- } \\
\text { rus; the disease was man- } \\
\text { made to enforce vaccination; } \\
\text { microchip implanting and in- } \\
\text { fertility claims }\end{array}$ & Low \\
\hline $\begin{array}{c}\text { Sharevski \& } \\
\text { Gover }(2021)^{35}\end{array}$ & USA & $\begin{array}{c}\text { January and } \\
\text { February } \\
2021 .\end{array}$ & $\begin{array}{c}\text { Cross-sectional } \\
\text { quasi-experimental } \\
\text { study }\end{array}$ & Twitter & $\begin{array}{c}304 \text { respond- } \\
\text { ents, } 18 \text { years } \\
\text { or older }\end{array}$ & $\begin{array}{l}\text { Exaggeration of rare side-ef- } \\
\text { fects of the COVID-19 vac- } \\
\text { cines }\end{array}$ & Low \\
\hline $\begin{array}{l}\text { Zhang et al } \\
(2021)^{36}\end{array}$ & China & $\begin{array}{c}\text { September } 1- \\
7,2020\end{array}$ & $\begin{array}{l}\text { Cross-sectional } \\
\text { study }\end{array}$ & $\begin{array}{l}\text { WeChat, } \\
\text { WeChat } \\
\text { moments, } \\
\text { Weibo, } \\
\text { TikTok } \\
\end{array}$ & \begin{tabular}{|}
2053 Chinese \\
factory work- \\
ers full-time \\
employees 18 \\
years or older
\end{tabular} & $\begin{array}{c}\text { Negative information about } \\
\text { Covid-19 vaccines }\end{array}$ & Low \\
\hline $\begin{array}{l}\text { Zhang et al } \\
(2021)^{37}\end{array}$ & China & $\begin{array}{c}\text { September } 1 \\
\text { to } 7,2020 .\end{array}$ & $\begin{array}{l}\text { Cross-Sectional } \\
\text { study }\end{array}$ & \begin{tabular}{|c|} 
WeChat, \\
WeChat \\
moments, \\
Weibo, \\
TikTok \\
\end{tabular} & $\begin{array}{c}2053 \text { Chinese } \\
\text { parents, } 18 \\
\text { years or older }\end{array}$ & $\begin{array}{c}\text { Negative information about } \\
\text { Covid-19 vaccines }\end{array}$ & Low \\
\hline $\begin{array}{c}\text { Costantino et al } \\
(2021)^{38}\end{array}$ & Italy & $\begin{array}{l}\text { December } \\
2020 \text { to } \\
\text { March } 2021\end{array}$ & $\begin{array}{l}\text { Cross-sectional } \\
\text { study }\end{array}$ & $\begin{array}{l}\text { Not speci- } \\
\text { fied }\end{array}$ & 363 adults & $\begin{array}{l}\text { Unfavourable information } \\
\text { about Covid-19 vaccines }\end{array}$ & Moderate \\
\hline $\begin{array}{c}\text { El-Far Cardo et } \\
\text { al }(2021)^{39}\end{array}$ & Germany & $\begin{array}{l}\text { August and } \\
\text { November } \\
2020\end{array}$ & $\begin{array}{l}\text { cross-sectional } \\
\text { study }\end{array}$ & $\begin{array}{l}\text { Facebook, } \\
\text { Twitter, } \\
\text { Telegram }\end{array}$ & 808 persons & $\begin{array}{c}\text { COVID-19 is not a health } \\
\text { threat. }\end{array}$ & Moderate \\
\hline $\begin{array}{l}\text { Jennings et al } \\
\quad(2021)^{40}\end{array}$ & UK & $\begin{array}{c}\text { Survey: } 12 \text { to } \\
18 \text { December } \\
2020 . \\
\text { Focus groups: } \\
30 \\
\text { November to } \\
7 \text { December } \\
2020\end{array}$ & $\begin{array}{c}\text { Cross-sectional } \\
\text { qualitative and } \\
\text { quantitative } \\
\text { (Mixed Method) }\end{array}$ & $\begin{array}{l}\text { Tik Tok, } \\
\text { Instagram, } \\
\text { Snapchat, } \\
\text { Twitter; } \\
\text { Facebook, } \\
\text { Youtube }\end{array}$ & \begin{tabular}{|c|}
1476 UK \\
adults partici- \\
pated in the \\
survey \\
\\
29 UK adults \\
participated in \\
the focus \\
groups
\end{tabular} & $\begin{array}{l}\text { Conspiracy theories } \\
\text { (not specified) }\end{array}$ & Moderate \\
\hline $\begin{array}{l}\text { Knights et al } \\
\quad(2021)^{41}\end{array}$ & $\begin{array}{l}\text { United } \\
\text { Kingdom }\end{array}$ & $\begin{array}{c}18 \text { June and } \\
30 \text { November } \\
2020\end{array} \mid$ & $\begin{array}{l}\text { Cross-sectional } \\
\text { qualitative study }\end{array}$ & $\begin{array}{c}\text { Not speci- } \\
\text { fied }\end{array}$ & \begin{tabular}{|c|}
64 Primary \\
Care Profes- \\
sionals and \\
administrative \\
staff, and 17 \\
recently-ar- \\
rived migrants \\
\end{tabular} & $5 \mathrm{G}$ conspiracy theory & Moderate \\
\hline $\begin{array}{l}\text { Berry et al } \\
(2021)^{42}\end{array}$ & USA & $\begin{array}{c}\text { December } 30 \\
2020, \text { to Janu- } \\
\text { ary } 15,2021\end{array}$ & $\begin{array}{l}\text { Qualitative obser- } \\
\text { vational study }\end{array}$ & $\begin{array}{l}\text { Not speci- } \\
\text { fied }\end{array}$ & $\begin{array}{c}193 \text { skilled } \\
\text { nursing facil- } \\
\text { ity workers }\end{array}$ & $\begin{array}{l}\text { Vaccines cause COVID-19; } \\
\text { microchip; the virus has been } \\
\text { around for a long time and } \\
\text { killed many people since } \\
\text { 1918; fear of racist motives } \\
\text { and the safety of the vac- } \\
\text { cines; the vaccines have foe- } \\
\text { tal cells from abortions }\end{array}$ & High \\
\hline
\end{tabular}




\begin{tabular}{|c|c|c|c|c|c|c|c|}
\hline $\begin{array}{c}\text { Choudhary et al } \\
(2021)^{43}\end{array}$ & India & $\begin{array}{c}\text { February 18 } \\
\text { to February } \\
28,2021\end{array}$ & $\begin{array}{c}\text { Cross-sectional } \\
\text { study }\end{array}$ & $\begin{array}{c}\text { Not speci- } \\
\text { fied }\end{array}$ & $\begin{array}{c}\text { 272 Indian } \\
\text { adults, 18 } \\
\text { years or older }\end{array}$ & COVID-19 is a conspiracy & High \\
\hline
\end{tabular}


Table 2: Studies in which data was collected from social media platforms $(\mathrm{n}=\mathbf{2 3})$

\begin{tabular}{|c|c|c|c|c|c|c|c|}
\hline Author (year) & Country & $\begin{array}{c}\text { Study Pe- } \\
\text { riod }\end{array}$ & $\begin{array}{c}\text { Study de- } \\
\text { sign }\end{array}$ & $\begin{array}{c}\text { Type of Social } \\
\text { Media }\end{array}$ & \begin{tabular}{|c|}
$\begin{array}{l}\text { Social Media or } \\
\text { population sam- } \\
\text { ple }\end{array}$ \\
\end{tabular} & Type of misinformation reported & Risk of bias (JBI) \\
\hline Chan et al $(2021)^{44}$ & $\begin{array}{l}\text { United } \\
\text { Kingdom }\end{array}$ & $\begin{array}{c}\text { December } \\
10,2020\end{array}$ & \begin{tabular}{|c|} 
Cross-sec- \\
tional (Social \\
media data \\
extraction)
\end{tabular} & YouTube & $\begin{array}{l}48 \text { COVID-19 vac- } \\
\text { cine-related videos } \\
\text { on YouTube }\end{array}$ & $\begin{array}{c}\text { Misinformation about the COVID-19 vac- } \\
\text { cines (not specified). Only } 2(4 \cdot 2 \%) \text { videos } \\
\text { made nonfactual claims. }\end{array}$ & Moderate \\
\hline $\begin{array}{l}\text { Alliheibi et al } \\
\quad(2021)^{45}\end{array}$ & $\begin{array}{l}\text { Saudi } \\
\text { Arabia }\end{array}$ & $\mid \begin{array}{c}\text { I5 Decem- } \\
\text { ber } 2020 \\
\text { to } 25 \text { May } \\
2021\end{array}$ & \begin{tabular}{|c|} 
Cross-sec- \\
tional (Social \\
media data \\
extraction)
\end{tabular} & Twitter & $\begin{array}{c}37,467 \text { tweets from } \\
23,748 \text { users }\end{array}$ & $\begin{array}{c}\text { COVID-19 vaccination is a cover for a plan } \\
\text { devised by Bill Gates to implant trackable } \\
\text { microchips to control people }\end{array}$ & High \\
\hline $\begin{array}{l}\text { Baines et al } \\
(2021)^{46}\end{array}$ & USA & $\begin{array}{l}20 \text { Novem- } \\
\text { ber } 2020, \\
\text { to } 6 \text { Janu- } \\
\text { ary } 2021\end{array}$ & $\begin{array}{c}\text { Cross-sec- } \\
\text { tional (Social } \\
\text { media data } \\
\text { extraction }\end{array}$ & Parler & $\begin{array}{c}400 \text { random par- } \\
\text { leys from a large } \\
\text { sample of } 7000 \\
\text { Parleys }\end{array}$ & $\begin{array}{c}\text { Sterilization possibilities for men and } \\
\text { women; COVID-19 vaccine to control the } \\
\text { population; Bill Gates and Anthony Fauci } \\
\text { had instigated measures (i.e., microchips and } \\
\text { enzymes in the vaccine) to control the popu- } \\
\text { lation through the administration of the } \\
\text { COVID-19 vaccine; governments and cer- } \\
\text { tain powerful individuals "planned" this } \\
\text { health crisis to vaccinate children without } \\
\text { parental consent as part of the new world or- } \\
\text { der to control future populations }\end{array}$ & High \\
\hline Basch et al $(2021)^{47}$ & 7 USA & $\begin{array}{c}\text { December } \\
2020\end{array}$ & \begin{tabular}{|c|} 
Cross-sec- \\
tional (Social \\
media data \\
extraction \\
\end{tabular} & TikTok & $\begin{array}{c}100 \text { videos studied } \\
\text { garnered } \\
35,338,600 \text { views }\end{array}$ & $\begin{array}{c}38 \text { videos discouraged the vaccine. } \\
3 \text { videos claimed that the vaccine is a hoax. }\end{array}$ & High \\
\hline $\begin{array}{l}\text { Boucher et al } \\
\qquad(2021)^{48}\end{array}$ & & $\begin{array}{c}\text { November } \\
19 \text { and } 26 \\
2020\end{array}$ & \begin{tabular}{|c|} 
Cross-sec- \\
tional (Social \\
media data \\
extraction
\end{tabular} & Twitter & $\begin{array}{c}\text { 636,516 English } \\
\text { and French Tweets }\end{array}$ & $\begin{array}{l}\text { COVID-19 vaccines are poison and the } \\
\text { mRNA technology has not been tested yet } \\
\text { and is harmful. }\end{array}$ & High \\
\hline $\begin{array}{l}\text { Chriss et al } \\
(2021)^{49}\end{array}$ & USA & $\begin{array}{l}\text { October } \\
2020 \text { to } \\
\text { January } \\
2021\end{array}$ & $\begin{array}{c}\text { Cross-sec- } \\
\text { tional (Social } \\
\text { media data } \\
\text { extraction }\end{array}$ & Twitter & 1110 tweets & $\begin{array}{l}\text { Misleading information that countered sci- } \\
\text { entific research about the vaccines; the gov- } \\
\text { ernment using vaccines to insert microchips } \\
\text { and control the population; the immune sys- } \\
\text { tem is stronger than the vaccines; race exter- } \\
\text { mination conspiracy that claims that the vac- } \\
\text { cine was created to "kill off [people of } \\
\text { color] POC." }\end{array}$ & High \\
\hline $\begin{array}{l}\text { Doyno et al } \\
(2021)^{50}\end{array}$ & USA & $\begin{array}{c}\text { January to } \\
\text { April } \\
2021 .\end{array}$ & $\begin{array}{c}\text { Quasi-exper- } \\
\text { imental }\end{array}$ & \begin{tabular}{|} 
YouTube, \\
Twitter, Face- \\
book, and In- \\
stagram
\end{tabular} & \begin{tabular}{|c|} 
Information cam- \\
paign with 79 \\
COVID-19 vac- \\
cine-related videos \\
in English, Can- \\
tonese, Spanish, \\
Mandarin, and \\
Polish \\
\end{tabular} & $\begin{array}{l}\text { Misinformation } \\
\text { (not specified) }\end{array}$ & High \\
\hline $\begin{array}{l}\text { Guntuku et al } \\
\quad(2021)^{51}\end{array}$ & USA & $\begin{array}{l}\text { December } \\
1,2020 \text { to } \\
\text { February } \\
28,2021\end{array}$ & $\begin{array}{c}\text { Cross-sec- } \\
\text { tional (Social } \\
\text { media data } \\
\text { extraction }\end{array}$ & Twitter & $\begin{array}{l}78.1 \text { million vac- } \\
\text { cine-related tweets }\end{array}$ & $\begin{array}{c}\text { Evangelical hubs posted conspiracy theories } \\
\text { about Bill Gates and China }\end{array}$ & High \\
\hline $\begin{array}{c}\text { Hernández-García } \\
\text { et al }(2021)^{52}\end{array}$ & Spain & $\begin{array}{l}9 \text { Febru- } \\
\text { ary } 2021\end{array}$ & \begin{tabular}{|c|} 
Cross-sec- \\
tional (Social \\
media data \\
extraction
\end{tabular} & YouTube & $\begin{array}{l}118 \text { YouTube vid- } \\
\text { eos }\end{array}$ & $\begin{array}{l}\text { Hoaxes and conspiracy theories } \\
\text { (not specified) }\end{array}$ & High \\
\hline $\begin{array}{l}\text { Herrera-Peco et al } \\
(2021)^{53}\end{array}$ & Spain & $\begin{array}{c}8 \text { to } 23 \\
\text { December } \\
2020\end{array}$ & \begin{tabular}{|} 
Cross-sec- \\
tional (Social \\
media data \\
extraction
\end{tabular} & Twitter & $\begin{array}{c}6080 \text { Twitter inter- } \\
\text { actions ( } \mathrm{n}=499 \text { of } \\
\text { those are single } \\
\text { tweets) }\end{array}$ & $\begin{array}{l}\text { Deny the existence of the virus; the vaccine } \\
\text { will modify the DNA of human beings; in- } \\
\text { dustry lobbies to kill the elderly and leave } \\
\text { the young with Bells syndrome. }\end{array}$ & High \\
\hline
\end{tabular}




\begin{tabular}{|c|c|c|c|c|c|c|c|}
\hline $\begin{array}{l}\text { Herrera-Peco et al } \\
\quad(2021)^{54}\end{array}$ & Spain & $\mid \begin{array}{c}14 \text { to } 28 \\
\text { December } \\
2020\end{array}$ & $\begin{array}{c}\text { Cross-sec- } \\
\text { tional (Social } \\
\text { media data } \\
\text { extraction }\end{array}$ & Twitter & $\begin{array}{c}5040 \text { Twitter users } \\
\text { participated, gener- } \\
\text { ating a total of } \\
1,664,261 \text { impres- } \\
\text { sions }\end{array}$ & $\begin{array}{c}\text { mRNA vaccines will produce changes in hu- } \\
\text { man DNA; government and pharmaceutical } \\
\text { industries are allies; adverse effects leading } \\
\text { to genocide }\end{array}$ & High \\
\hline $\begin{array}{l}\text { Hughes et al } \\
(2021)^{55}\end{array}$ & USA & $\begin{array}{c}\text { October } \\
2020 \text { to } \\
\text { November } \\
2020\end{array}$ & \begin{tabular}{|c|} 
Cross-sec- \\
tional (Social \\
media data \\
extraction + \\
modelling)
\end{tabular} & $\begin{array}{l}\text { Facebook, } \\
\text { Twitter, } \\
\text { YouTube, and } \\
\text { Instagram }\end{array}$ & \begin{tabular}{|} 
Using hashtag and \\
keyword searchers, \\
a team of subject \\
matter experts \\
identified 20 chan- \\
nels (i.e., bounded \\
sources of content, \\
such as a social \\
media account), \\
which appeared to \\
contain a high de- \\
gree of anti-vac- \\
cine content and/or \\
COVID denialism.
\end{tabular} & \begin{tabular}{|} 
Corrupt Elites; physical deformities; mental \\
illness; microchips that violate your auton- \\
omy/privacy; the people who intentionally \\
created the COVID vaccine are shadowy \\
and suspicious
\end{tabular} & High \\
\hline Islam et al $(2021)^{56}$ & Australia & \begin{tabular}{|c}
31 De- \\
cember \\
$2019-30$ \\
November \\
2020
\end{tabular} & $\begin{array}{c}\text { Cross-sec- } \\
\text { tional (Social } \\
\text { media data } \\
\text { extraction }\end{array}$ & $\begin{array}{l}\text { Facebook, } \\
\text { YouTube, and } \\
\text { Twitter }\end{array}$ & $\begin{array}{c}637 \text { news articles, } \\
\text { social media narra- } \\
\text { tives, online re- } \\
\text { ports, and/or blogs } \\
\text { spread on social } \\
\text { media }\end{array}$ & $\begin{array}{c}\text { Daughter of the Russian president had died } \\
\text { after receiving the second dose of COVID- } \\
19 \text { vaccine; children and soldiers dying after } \\
\text { receiving the vaccine in multiple countries; } \\
\text { conspiracy theory about Bill Gates; COVID- } \\
19 \text { vaccine can monitor the human popula- } \\
\text { tion and take over the world; COVID-19 } \\
\text { vaccines contain a microchip through which } \\
\text { biometric data could be collected, and large } \\
\text { businesses could send signals to the chips } \\
\text { using 5G networks; crucial phases of the } \\
\text { clinical trials were skipped; COVID-19 vac- } \\
\text { cine contains cells from aborted foetus or } \\
\text { genes from pigs }\end{array}$ & High \\
\hline Kwok et al $(2021)^{57}$ & Australia & \begin{tabular}{|c|} 
January \\
22 and \\
October \\
20,2020
\end{tabular} & \begin{tabular}{|c|} 
Cross-sec- \\
tional (Social \\
media data \\
extraction
\end{tabular} & Twitter & $\begin{array}{c}31,100 \text { COVID-19 } \\
\text { vaccine-related } \\
\text { tweets }\end{array} \mid$ & $\begin{array}{c}\text { Conspiracy theories such as the "mark of the } \\
\text { beast" and microchips in vaccines. }\end{array}$ & High \\
\hline $\begin{array}{l}\text { Larrondo-Ureta } \\
\qquad(2021)^{58}\end{array}$ & $\begin{array}{l}\text { Spanish- } \\
\text { speaking } \\
\text { countries }\end{array}$ & $\begin{array}{c}\text { December } \\
2020 \text { and } \\
\text { February } \\
2021\end{array}$ & $\begin{array}{c}\text { Cross-sec- } \\
\text { tional (Social } \\
\text { media data } \\
\text { extraction }\end{array}$ & Twitter & $\begin{array}{c}62,045 \text { tweets and } \\
258,843 \text { retweets }\end{array}$ & $\begin{array}{l}\text { Anti-vaccine discourse } \\
\quad \text { (not specified) }\end{array}$ & High \\
\hline Liu \& Liu $(2021)^{59}$ & $\begin{array}{l}\text { English- } \\
\text { speaking } \\
\text { countries }\end{array}$ & $\mid \begin{array}{c}\text { November } \\
1-22 \\
2020\end{array}$ & \begin{tabular}{|c|} 
Cross-sec- \\
tional (Social \\
media data \\
extraction
\end{tabular} & Twitter & $\begin{array}{c}5000 \text { COVID-19 } \\
\text { vaccine-related } \\
\text { tweets, which were } \\
\text { posted by } 4796 \\
\text { unique users. } \\
\end{array}$ & $\begin{array}{l}\text { Microchips; alters DNA; women become } \\
\text { sterile }\end{array}$ & High \\
\hline $\begin{array}{l}\text { Melton et al } \\
\quad(2021)^{60}\end{array}$ & USA & $\begin{array}{l}\text { Dec 1, } \\
2020 \text {, to } \\
\text { May } 15 \\
2021\end{array}$ & $\begin{array}{c}\text { Cross-sec- } \\
\text { tional (Social } \\
\text { media data } \\
\text { extraction }\end{array}$ & Reddit & $\begin{array}{c}13 \text { Reddit commu- } \\
\text { nities }\end{array}$ & Misinformation about side-effects & High \\
\hline $\begin{array}{l}\text { Pascual-Ferra } \\
\quad(2021)^{61}\end{array}$ & USA & $\begin{array}{c}\text { December } \\
29,2019, \\
\text { to January } \\
2,2021\end{array}$ & $\mid \begin{array}{c}\text { Cross-sec- } \\
\text { tional (Social } \\
\text { media data } \\
\text { extraction }\end{array}$ & $\begin{array}{l}\text { Facebook, In- } \\
\text { stagram, Red- } \\
\text { dit, YouTube }\end{array}$ & $\begin{array}{c}\text { Peaks and interac- } \\
\text { tions }\end{array}$ & $\begin{array}{c}\text { Viral video of a nurse fainting after vaccine } \\
\text { uptake. } \\
\begin{array}{c}\text { Misinformation about COVID-19 vaccines } \\
\text { (not specified) }\end{array}\end{array}$ & High \\
\hline $\begin{array}{l}\text { Rotolo et al } \\
(2021)^{62}\end{array}$ & USA & \begin{tabular}{|} 
March 19, \\
2020 , and \\
June 16, \\
2021
\end{tabular} & \begin{tabular}{|} 
Quasi-Exper- \\
imental \\
(Social me- \\
dia data ex- \\
traction
\end{tabular} & $\begin{array}{l}\text { Facebook, } \\
\text { Twitter, and } \\
\text { Instagram }\end{array}$ & $\begin{array}{l}\text { Aim: Share } 49 \text { in- } \\
\text { fographics to } \\
\text { counter vaccine } \\
\text { hesitancy. }\end{array}$ & COVID-19 myths & High \\
\hline
\end{tabular}




\begin{tabular}{|c|c|c|c|c|c|c|c|}
\hline $\begin{array}{l}\text { Savolainen } \\
(2021)^{63}\end{array}$ & Finland & $\begin{array}{c}\text { February } \\
2021\end{array}$ & $\begin{array}{c}\text { Cross-sec- } \\
\text { tional (Social } \\
\text { media data } \\
\text { extraction) }\end{array}$ & $\begin{array}{c}\text { Reddit, from } \\
\text { the subreddit } \\
\text { VaxxHappened }\end{array}$ & $\begin{array}{c}40 \text { threads con- } \\
\text { tained in total } 1877 \\
\text { messages }\end{array}$ & $\begin{array}{c}\text { Misinformation about COVID-19 vaccines } \\
\text { (not specified) }\end{array}$ & High \\
\hline $\begin{array}{l}\text { Sobkowicz \& Sob- } \\
\text { kowicz }(2021)^{64}\end{array}$ & $\begin{array}{c}\text { USA and } \\
\text { Poland }\end{array}$ & $\begin{array}{l}1 \text { March } \\
2021\end{array}$ & \begin{tabular}{|c|} 
Cross-sec- \\
tional (Social \\
media data \\
extraction
\end{tabular} & $\begin{array}{l}\text { Reddit and In- } \\
\text { teria }\end{array}$ & $\begin{array}{c}\text { Reddit and Interia } \\
\text { Anti-vaccine } \\
\text { groups }\end{array}$ & $\begin{array}{l}\text { Anti-vaccination discussions about COVID- } \\
19 \text { vaccines }\end{array}$ & High \\
\hline $\begin{array}{l}\text { Thelwall et al } \\
\qquad(2021)^{65}\end{array}$ & $\begin{array}{c}\text { United } \\
\text { Kingdom }\end{array}$ & $\begin{array}{l}10 \text { March } \\
\text { to } 5 \text { De- } \\
\text { cember } \\
2020 .\end{array}$ & $\begin{array}{c}\text { Cross-sec- } \\
\text { tional (Social } \\
\text { media data } \\
\text { extraction }\end{array}$ & Twitter & $\begin{array}{l}446 \text { vaccine-hesi- } \\
\text { tant Covid-19 } \\
\text { tweets in English }\end{array}$ & $\begin{array}{c}\text { Deep state conspiracy; depopulation; micro- } \\
\text { chips; Bill Gates; fearing that people of col- } \\
\text { our are at risk for experimentation - moti- } \\
\text { vated by the infamous US federal govern- } \\
\text { ment Tuskegee Syphilis study ending in } \\
1972 \text { that secretly experimented on poor Af- } \\
\text { rican American men }\end{array}$ & High \\
\hline $\begin{array}{l}\text { Wawrzuta et al } \\
\qquad(2021)^{66}\end{array}$ & Poland & $\begin{array}{c}1 \text { Novem- } \\
\text { ber } 2020 \\
\text { and } \\
1 \text { May } \\
2021 \\
\end{array}$ & $\begin{array}{c}\text { Cross-sec- } \\
\text { tional (Social } \\
\text { media data } \\
\text { extraction }\end{array}$ & Facebook & $\begin{array}{l}3414 \text { Facebook } \\
\text { comments }\end{array}$ & $\begin{array}{c}\text { The vaccine was created only for the profit } \\
\text { of pharmaceutical companies; conspiracy } \\
\text { theories, hidden vaccine effects (e.g., chips); } \\
\text { the vaccine will be dangerous to health; the } \\
\text { vaccine has existed before the COVID-19 } \\
\text { pandemic }\end{array}$ & High \\
\hline
\end{tabular}

We grouped the studies into two major categories according to data sampling: One group gathered data through surveys, interviews, or focus groups (Table 1). The other group gathered data from social media (Table 2). The largest total population sample in the first group of 22 studies seen in Table 1 came from Europe with 27975 respondents in total. European countries were represented by Poland, ${ }^{26}$ Italy, ${ }^{38}$ Norway, ${ }^{28}$ Germany, ${ }^{39}$ Slovenia, ${ }^{32}$ and the UK. The UK was represented with 9538 people from four studies..$^{23,27,40,41}$ The US sample size amounted to 12759 people from four studies. ${ }^{25,31,35,42}$ Middle Eastern countries were represented through studies from Kuwait, ${ }^{22}$ Jordan, ${ }^{24,33}$ and Saudi Arabia ${ }^{34}$ and counted for 9313 people of the total sample. A total of 7082 participants came from Asian countries such as China, ${ }^{36,37}$ India, ${ }^{43}$ and Turkey. ${ }^{30}$ Only one included study in our review came from an African country. ${ }^{29}$ Feil! Bokmerke er ikke definert. The study was from Uganda and included 600 participants. There were no studies from Middle- or South America or Pacific Island countries and Australia. All of the respondents were described as adults or older than 18 years except for two US studies where the participants were 65 years or older. ${ }^{25,31}$ Another exception was one study from Slovenia where participants 15 years or older were included. ${ }^{32}$ The participants' occupation was mentioned in some studies as being medical student, ${ }^{29}$ university student, ${ }^{33}$ Medicare enrollee, ${ }^{25}$ Medicare beneficiary, ${ }^{31}$ factory worker, ${ }^{36,37}$ or skilled nursing facility worker. ${ }^{42}$ One Polish study focused on cancer patients. ${ }^{26}$ In one of the few cross-sectional qualitative studies by Knights and colleagues, primary care professionals and recently arrived immigrants were interviewed. ${ }^{41}$ Gender has not been a focal point in any of the 45 included studies. 
In the second group, where data was gathered from social media platforms, we found 23 studies. Ten studies harvested data from Twitter. Five of these examined English language tweets, ${ }^{49,51,57,59,65}$ three studies gathered tweets in Spanish, ${ }^{53,54,58}$ one study examined tweets in both English and French ${ }^{49}$ and one study examined Arabic tweets. ${ }^{45}$ Five studies gathered data from several social media platforms, most commonly combining Twitter, Facebook, Instagram, and YouTube. ${ }^{50,55,56,61,62}$ These studies looked at English language messages or videos. Two studies examined English language messages or discourse on Reddit. ${ }^{60,63}$ One study looked at anti-vaccine discussions on both Reddit and the Polish social media platform Interia. ${ }^{64}$ Two studies examined YouTube videos in English ${ }^{44}$ and in Spanish. ${ }^{52}$ One US study looked at English language TikTok messages ${ }^{47}$ and another US study examined English language messages on the American social media platform Parler. ${ }^{46}$ Finally, one Polish study examined Facebook comments. ${ }^{66}$

Many of the studies did not name social media platforms in the first group (Table 1), but rather discussed social media platforms in general. However, some studies did specify which social media platforms they were assessing. The studies with low risk of bias that specified the social media platform they were assessing focused on Twitter only ${ }^{35}$ and several platforms, such as Facebook, Instagram, YouTube, WhatsApp, WeChat, Weibo, and TikTok. ${ }^{30,34,36,37}$ Among the studies that collected data from social media (Table 2), ten studies extracted data from Twitter, ${ }^{45,48,49,51,53,54,57-59,65}$ two from YouTube, ${ }^{44,52}$ one from TikTok, ${ }^{47}$ one from Reddit, ${ }^{60}$ one from Parler, ${ }^{46}$ and one from Facebook. ${ }^{66}$ Seven studies that collected data from social media utilized two or more platforms. ${ }^{50,55,56,61-64}$ Figure 2 summarizes the types of social media platforms used in the 45 included studies. 
14

\begin{tabular}{|c|c|}
\hline Social Media Platforms & Number \\
\hline Twitter & 18 \\
\hline (f) Facebook & 9 \\
\hline YouTube & 7 \\
\hline (0) Instagram & 6 \\
\hline TikTok & 4 \\
\hline Reddit & 4 \\
\hline WeChat & 2 \\
\hline Wechat Moments & 2 \\
\hline Weibo & 2 \\
\hline (2) WhatsApp & 2 \\
\hline Interia & 1 \\
\hline Parler & 1 \\
\hline Snapchat & 1 \\
\hline ( $)$ Telegram & 1 \\
\hline
\end{tabular}

Figure 2: Social Media Platforms 
Figure 3 gives an overview of three overarching themes of misinformation identified from the thematic analysis, and examples of topics of misinformation within the three themes.



Figure 3: Types of misinformation about the COVID-19 vaccine on social media platforms

Of the 45 included studies, 18 studies reported misinformation across all three categories. ${ }^{24-26,32-}$ $34,42,45,46,48,49,53-56,59,65,66$ Nine studies reported on conspiracies only. $23,27,30,40,41,43,51,52,57$ Six studies were 
16

concerned specifically with medical misinformation. ${ }^{22,31,35,39,60,61} 12$ studies reported on COVID-19 vaccine misinformation or anti-vaccine discourse without going into further detail. ${ }^{28,32,36-38,44,47,50,58,62-64}$

We identified 19 studies that made assumptions about the effects of social media misinformation on vaccine hesitancy (Table 4). The evaluation of the certainty of evidence of these 19 studies that measured the "Association between social media misinformation and COVID-19 vaccine hesitancy" was classified as moderate or low-moderate according to GRADE in two cases. ${ }^{35,62}$ For the rest of the studies, the certainty of the evidence according to GRADE was considered low or very low (see Table 3).

The two studies with a higher certainty of the evidence had an experimental design. Rotolo and colleagues ${ }^{62}$ aimed at developing and distributing infographics that addressed COVID-19 vaccine hesitancy and misinformation. Although their infographics reached thousands of people, they were unable to conclude the impact on vaccine hesitancy. Sharevski \& Gover ${ }^{35}$ analyzed the perceived accuracy of COVID-19 vaccine Tweets when they were moderated by smart device technology that Twitter applies to COVID-19 misinformation. The results from the 304 participants suggested that the vaccination hesitant users ignored warnings as long as the Tweets aligned with their personal beliefs.

Allington and colleagues ${ }^{23}$ analysed findings from an online survey conducted with a sample of 4343 UK adults. They found a positive correlation between trust in social media and vaccine hesitancy, and the strongest link was found concerning YouTube and Facebook. Conspiracy suspicions about COVID-19 and general vaccine attitudes appeared to be uniquely predictive, jointly explaining $35 \%$ of the variance. Boucher and colleagues ${ }^{48}$ analysed 636,516 English and French Tweets. 23.4\% $(n=146,191)$ of conversations on Twitter during the study period could be directly attributed to vaccine hesitancy. A British study ${ }^{27}$ of 5114 adults found that social media dependence and high levels of conspiracy mentality were most likely to be associated with online discouragement of vaccination. In a study of 4571 Norwegian adults, individuals who preferred social media platforms had a near twofold (i.e., 1.64) odds of being hesitant toward COVID-19 vaccination compared to those preferring sourceverified media platforms. ${ }^{28}$ In addition, those who held the belief in the superiority of natural immunity over vaccination were more vaccine-hesitant $(\mathrm{OR}=2 \cdot 663 ; 95 \% \mathrm{CI}=2 \cdot 350,3 \cdot 028, \mathrm{p}<0 \cdot 001)$. Petravic and colleagues ${ }^{32}$ asked 12,042 Slovenian residents about attitudes on COVID-19 vaccines. Those who 
trusted alternative media sources and alternative explanations on social media were more vaccine-hesitant.

12 studies $^{24-26,30,31,33,36-40,56}$ discussed social media misinformation, vaccine uptake, and vaccine intentions. In a study of 646 Jordanians $^{24}$, social media were found to affect the decision to get a vaccine $(\mathrm{OR}=1 \cdot 21 ; 95 \% \mathrm{CI}=1 \cdot 04,1 \cdot 41 ; \mathrm{p}=0.012)$ and $43.8 \%(\mathrm{n}=238)$ believed that it was unsafe while $15.6 \%(n=101)$ believed that the vaccination was part of secret research. Bhagianadh \& Arora ${ }^{25}$ examined the prevalence and predictors of vaccine hesitancy among 5784 older (>65 years) US citizens. Those depending on social media as the main source of information on COVID-19 expressed higher negative vaccine intent $(\mathrm{OR}=3 \cdot 36 ; 95 \% \mathrm{CI}=1 \cdot 44,7 \cdot 82)$. Among those who expressed a negative vaccine intent, $40 \%(\mathrm{n}=298)$ expressed no trust in the government and $10 \%(\mathrm{n}=74)$ said that the vaccines cause COVID-19. In a Polish study by Brodiziak and colleagues ${ }^{26}$ of 635 adult cancer patients, they found that the use of social media as an information source was associated with unwillingness to be vaccinated $(\mathrm{OR}=1 \cdot 42 ; 95 \% \mathrm{CI}=0 \cdot 72,2 \cdot 80) .68 \%(\mathrm{n}=432)$ used social media every day. $37.6 \%(n=239)$ said that they were afraid of the composition of the vaccine, $7.7 \%(n=49)$ believed that the vaccines contained bodies of aborted children, and $6.6 \%(n=42)$ believed that COVID-19 did not exist. In an Italian study of 363 adults, ${ }^{38} 71.4 \%(n=60)$ answered that unfavourable information about COVID-19 vaccines obtained from the Internet/social media/media was associated with the decision to not take the vaccine. El-Far Cardo and colleagues ${ }^{39}$ asked 808 Germans to complete a questionnaire about media preference and vaccine willingness. Using social media as an information source was negatively associated with getting vaccinated against COVID-19 ( $<<0.01)$. Believing that COVID-19 is not dangerous was also associated with an unwillingness to get vaccinated. A UK study by Jennings and colleagues ${ }^{40}$ found that users of Instagram, Snapchat, and TikTok were less likely to express willingness to be vaccinated. However, YouTube users were the most vaccine-hesitant. Holding conspiracy beliefs was also a significant predictor of vaccine hesitancy. The study involved 1476 UK adults who participated in a survey and 29 in focus groups. Karabela and colleagues ${ }^{30}$ investigated attitudes towards vaccines and their trust in information sources among 1216 Turkish adults. The participants who stated that they would have the COVID-19 vaccine were less likely to trust social media sites such as Facebook, Twitter, and Instagram ( $<$ 0.005). On the other hand, those who were undecided were more likely to trust YouTube as a reliable source of information. The researchers also found a positive 
relationship between attitudes towards the COVID-19 vaccines and conspiracy theories $(r=0 \cdot 214)$. Liu and $\mathrm{Liu}^{59}$ examined 5000 COVID-19 vaccine-related tweets and found that 182 tweets contained negative behavioural intentions meaning they would not choose to get vaccinated, and 97 tweets were labelled with positive behavioural intentions. Park and colleagues ${ }^{31}$ examined how the source of information shaped perceptions of COVID-19 severity and the likelihood of COVID-19 vaccine uptake among 6478 Medicare beneficiaries. Those relying on social media had the lowest levels in all measures. The likelihood of COVID-19 vaccine uptake was significantly lower among those relying on

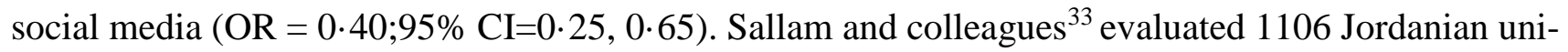
versity students' COVID-19 vaccine acceptance and examined the association between vaccine conspiracy beliefs and vaccine hesitancy. The lowest rate of intention to get the vaccine was among those who depended on social media platforms (19.8\%) compared to those who trusted doctors, scientists, and scientific journals $(47 \cdot 2 \%, \mathrm{p}<0 \cdot 001)$. The researchers also found that beliefs in conspiracy theories were correlated with reluctance to take the vaccine $(\mathrm{p}<0 \cdot 001)$. In a Chinese study of 2053 factory workers and their behavioural intentions to receive COVID-19 vaccination, Zhang and colleagues ${ }^{36}$ found that a higher frequency of exposure to positive information on social media related to COVID19 vaccination was associated with a higher intention to receive a COVID-19 vaccination at the market rate $(\mathrm{AOR}=1 \cdot 53 ; 95 \% \mathrm{CI}=1 \cdot 39,1 \cdot 70)$ or a free vaccination $(\mathrm{AOR}=1 \cdot 52 ; 95 \% \mathrm{CI} 1 \cdot 35,1 \cdot 71)$. Zhang and colleagues ${ }^{37}$ also investigated the parental acceptability of free COVID-19 vaccination for children under the age of 18 in China. The sample was the same as above, as this was a secondary analysis of a closed online survey among the 2053 factory workers. Higher exposure to positive information related to COVID-19 vaccination on social media was associated with higher parental acceptability of COVID-19 vaccination $(\mathrm{AOR}=1 \cdot 35 ; 95 \% \mathrm{CI}=1 \cdot 17,1 \cdot 56)$. Higher exposure to negative information related to COVID-19 vaccination was negatively associated with the dependent variable (AOR 0.85; $95 \% \mathrm{CI}=0.74,0.99)$.

Table 3: Studies in which the effect of social media misinformation on vaccine hesitancy is measured or discussed $(\mathbf{n}=19)$.

\begin{tabular}{|c|c|c|}
\hline Author (year) & Reported effect of misinformation & $\begin{array}{c}\text { Certainty of evidence } \\
\text { (GRADE) }\end{array}$ \\
\hline & $\begin{array}{c}\text { Amazon Alexa was not able to dispel any } \\
\text { biases that were rooted in personal beliefs. }\end{array}$ & Moderate \\
$\begin{array}{c}\text { One's hesitancy from COVID-19 vaccina- } \\
\text { Sharevski \& Gover sufficed for biased perception of the in- } \\
(2021)^{35}\end{array}$ & $\begin{array}{c}\text { formation from Alexa despite any labelling } \\
\text { as long as the Tweets echoed their sceptic } \\
\text { outlook on the whole COVID-19 vaccina- } \\
\text { tion effort. }\end{array}$ & \\
\hline
\end{tabular}




\begin{tabular}{|c|c|c|}
\hline Rotolo et al (2021)62 & $\begin{array}{c}\text { Each infographic reached thousands to tens } \\
\text { of thousands of people. We do not know } \\
\text { whether those who viewed these in- } \\
\text { fographics changed their perspective on } \\
\text { vaccination, so we are unable to conclude } \\
\text { their impact on vaccine hesitancy based on } \\
\text { this study alone. }\end{array}$ & Low-Moderate \\
\hline $\begin{array}{l}\text { Allington et al } \\
(2021)^{23}\end{array}$ & $\begin{array}{c}\text { Informational reliance on all social media } \\
\text { platforms was positively correlated with } \\
\text { vaccine hesitancy, this correlation was } \\
\text { strongest concerning Facebook and } \\
\text { YouTube (RS }=0 \cdot 15 \text { and } \mathrm{RS}=0 \cdot 18, \text { re- } \\
\text { spectively). } \\
\text { Coronavirus conspiracy suspicions and gen- } \\
\text { eral vaccine attitudes appear uniquely pre- } \\
\text { dictive, jointly explaining } 35 \% \text { of variance. }\end{array}$ & Low \\
\hline $\begin{array}{c}\text { Bhagianadh \& Arora } \\
(2021)^{25}\end{array}$ & \begin{tabular}{|c|} 
Those depending on social media as the \\
main source of information on COVID-19 \\
expressed higher negative vaccine intent \\
$(\mathrm{OR}=3 \cdot 36 ; 95 \% \mathrm{CI}=1 \cdot 44,7 \cdot 82)$. \\
Among those who expressed a negative \\
vaccine intent, $40 \%(\mathrm{n}=298)$ expressed no \\
trust in government, $10 \%(\mathrm{n}=74)$ said that \\
the vaccines cause COVID-19.
\end{tabular} & Low \\
\hline $\begin{array}{l}\text { Boucher et al } \\
(2021)^{48}\end{array}$ & $\begin{array}{c}\text { The study showed two clusters opposite to } \\
\text { these vaccine acceptant clusters exhibiting } \\
\text { more vaccine-hesitant narratives. There } \\
\text { were } 23 \cdot 4 \%(\mathrm{n}=146,191) \text { of conversations } \\
\text { on Twitter during this period of observation } \\
\text { that can be directly attributed to vaccine } \\
\text { hesitancy. }\end{array}$ & Low \\
\hline $\begin{array}{l}\text { Chadwick et al } \\
\qquad(2021)^{27}\end{array}$ & $\begin{array}{c}\text { Combinations of news avoidance and high } \\
\text { levels of the news-finds-me attitude and so- } \\
\text { cial media dependence and high levels of } \\
\text { conspiracy mentality are most likely to be } \\
\text { associated with online discouragement of } \\
\text { vaccination. }\end{array}$ & Low \\
\hline Jennings et al (2021 $_{40}$ & $\begin{array}{c}\text { Holding conspiracy beliefs is a significant } \\
\text { predictor of vaccine hesitancy. } \\
\text { In the bivariate analysis, there is some sup- } \\
\text { port for a relationship between social media } \\
\text { use Snapchat, TikTok, YouTube, and Insta- } \\
\text { gram) and increased vaccine hesitancy. } \\
\text { YouTube users were significantly less will- } \\
\text { ing to be vaccinated, with a two-thirds like- } \\
\text { lihood of vaccine willingness compared to } \\
\text { non-users. }\end{array}$ & Low \\
\hline Liu \& Liu $(2021)^{59}$ & $\begin{array}{c}279 \text { tweets stated their behavioural inten- } \\
\text { tions. A total of } 97 \text { tweets were labelled } \\
\text { with positive behavioural intentions, while } \\
182 \text { tweets contained negative behavioural } \\
\text { intentions. }\end{array}$ & Low \\
\hline Park et al $\left(2021^{28}\right.$ & $\begin{array}{l}\text { The study found that social media depend- } \\
\text { ence and high levels of conspiracy mental- } \\
\text { ity were most likely to be associated with } \\
\text { online discouragement of vaccination. } \\
\text { The likelihood of COVID-19 vaccine up- } \\
\text { take was significantly lower among those } \\
\text { relying on social media (OR }=0.40 ; 95 \% \\
\text { CI }=0 \cdot 25,0 \cdot 65)\end{array}$ & Low \\
\hline Zhang et al $(2021)^{36}$ & \begin{tabular}{|} 
Regarding social media influence, higher \\
frequency of exposure to positive infor- \\
mation related to COVID-19 vaccination \\
was associated with a higher intention to re- \\
ceive a COVID-19 vaccination at market \\
rate (AOR $1 \cdot 53 ; 95 \%$ CI $1 \cdot 39-1 \cdot 70$ ) or a \\
\end{tabular} & Low \\
\hline
\end{tabular}




\begin{tabular}{|c|c|c|}
\hline & $\begin{array}{c}\text { free vaccination (AOR } 1 \cdot 52 ; 95 \% \text { CI } 1 \cdot 35- \\
1 \cdot 71) .\end{array}$ & \\
\hline Zhang et al $(2021)^{37}$ & \begin{tabular}{|c|} 
Higher exposure to positive information re- \\
lated to COVID-19 vaccination on social \\
media was associated with higher parental \\
acceptability of COVID-19 vaccination \\
(AOR $=1 \cdot 35 ; 95 \%$ CI $=1 \cdot 17-1 \cdot 56)$. Higher \\
exposure to negative information related to \\
COVID-19 vaccination was negatively as- \\
sociated with the dependent variable \\
$(\mathrm{AOR}=0 \cdot 85 ; 95 \% \mathrm{CI}=0 \cdot 74-0 \cdot 99)$.
\end{tabular} & Low \\
\hline $\begin{array}{l}\text { Aloweidi et al } \\
\quad(2021)^{24}\end{array}$ & $\begin{array}{c}\text { The effect of social media }(\mathrm{OR}=1 \cdot 21 ; 95 \% \\
\mathrm{CI}=1 \cdot 04,1 \cdot 41 ; \mathrm{p}=0 \cdot 012) \text { were signifi- } \\
\text { cantly associated with the willingness to } \\
\text { take COVID-19 vaccine. } \\
\text { Circulated information about COVID-19 } \\
\text { vaccines on social media platforms that } \\
\text { they believed in: It is unsafe } \mathrm{n}=283 \\
(43.8 \%) \text {; Effect of the vaccines on a genetic } \\
\text { level } \mathrm{n}=87(13 \cdot 5 \%) \text {; Causes chronic ill- } \\
\text { nesses } \mathrm{n}=60(9 \cdot 3 \%) ; \text { May lead to infertility } \\
\mathrm{n}=43(6 \cdot 7) ; \text { Can affect their offspring } \mathrm{n}=56 \\
(8 \cdot 7 \%) \text { Toxic heavy metals and neurotoxic } \\
\text { materials } \mathrm{n}=47(7 \cdot 3 \%) ; \text { It is a part of a se- } \\
\text { cret research } 101(15 \cdot 6 \%)\end{array}$ & Very Low-Low \\
\hline $\begin{array}{l}\text { Brodiziak et al } \\
\quad(2021)^{26}\end{array}$ & $\begin{array}{c}\mathrm{N}=432(68 \%) \text { used social media every day. } \\
\text { Unwilling to Vaccinate against COVID-19: } \\
\text { Social media as a source of information } \\
\text { about vaccinations (OR=1.42; } 95 \% \text { CI= } \\
0 \cdot 72,2 \cdot 80) \text {. Not a significant predictor } \\
\text { Attitudes to COVID-19 vaccines: } \\
\text { Afraid of the vaccine's side effects } n=284 \\
(44.7 \%) \text {; afraid of the composition of the } \\
\text { vaccine } n=239(37 \cdot 6 \%) \text {; contains bodies of } \\
\text { aborted children } n=49(7 \cdot 7 \%) \text {; Coronavirus } \\
\text { does not exist } n=42(6 \cdot 6 \%)\end{array}$ & Very Low-Low \\
\hline $\begin{array}{l}\text { Ebrahimi et al } \\
\quad(2021)^{28}\end{array}$ & $\begin{array}{c}\text { Individuals with a preference for social me- } \\
\text { dia platforms as compared to those prefer- } \\
\text { ring source-verified media platforms had a } \\
\text { near } 2 \text { fold (i.e., } 1.64) \text { odds of being hesitant } \\
\text { toward vaccination. } \\
\text { Belief in superiority of natural immunity: } \\
\mathrm{OR}=2 \cdot 663(95 \% \text { CI }=2 \cdot 350,3 \cdot 028) \mathrm{p}<0 \cdot 001\end{array}$ & Very Low-Low \\
\hline $\begin{array}{l}\text { El-Far Cardo et al } \\
\qquad\left(2021^{39}\right.\end{array}$ & $\begin{array}{c}\text { Factors that were negatively associated to } \\
\text { get vaccinated were using social media in } \\
\text { general as an information source about } \\
\text { COVID-19 }(\mathrm{p}<0 \cdot 01) \text {, and the use of Face- } \\
\text { book ( } \mathrm{p}<0 \cdot 05) \text { or Telegram }(\mathrm{p}<0 \cdot 05) \text {. } \\
\text { However, using Twitter was not signifi- } \\
\text { cantly associated with adverse vaccination } \\
\text { intentions }(\mathrm{p}<0 \cdot 56) \text {. } \\
\text { Believing that COVID-19 is not dangerous } \\
\text { was associated with unwillingness to get } \\
\text { vaccinated. }\end{array}$ & Very Low-Low \\
\hline $\begin{array}{l}\text { Petravic et al } \\
\quad(2021)^{32}\end{array}$ & $\begin{array}{c}\text { Those who trusted alternative media } \\
\text { sources (alternative explanations on social } \\
\text { media) and had a distrust of the government } \\
\text { were more vaccine-hesitant. }\end{array}$ & Very Low-Low \\
\hline Sallam et al $(2021)^{33}$ & \begin{tabular}{|c|} 
The lowest rate of intention to get the vac- \\
cine was among those who depended on so- \\
cial media platforms $(19 \cdot 8 \%)$ compared to \\
dependence on medical doctors, scientists, \\
and scientific journals $(47 \cdot 2 \%, p<0 \cdot 001$ ). \\
Conspiracy beliefs were evaluated using the \\
validated Vaccine Conspiracy Belief Scale \\
(VCBS), with higher scores implying
\end{tabular} & Very Low-Low \\
\hline
\end{tabular}




\begin{tabular}{|c|c|c|}
\hline & \begin{tabular}{|c|} 
embrace of conspiracies. A significantly \\
higher VCBS score was correlated with re- \\
luctance to get the vaccine $(\mathrm{p}<0 \cdot 001)$
\end{tabular} & \\
\hline $\begin{array}{l}\text { Costantino et al } \\
\quad(2021)^{38}\end{array}$ & $\begin{array}{l}71.4 \%(\mathrm{n}=60) \text { responded that unfavourable } \\
\text { information about COVID-19 vaccines ob- } \\
\text { tained from the Internet/social media/media } \\
\text { was associated with the decision to not take } \\
\text { the vaccine. }\end{array}$ & Very Low \\
\hline $\begin{array}{l}\text { Karabela et al } \\
\qquad(2021)^{30}\end{array}$ & $\begin{array}{c}\text { Although the correlation was not signifi- } \\
\text { cant, of the participants, those who consid- } \\
\text { ered having vaccination mostly trusted } \\
\text { YouTube as their source of information. On } \\
\text { the other hand, the participants who stated } \\
\text { that they would have the COVID-19 vac- } \\
\text { cine did not trust social media sites such as } \\
\text { Facebook, Twitter, and Instagram (p < } \\
0 \cdot 005) \\
\text { There was a positive and low-level relation- } \\
\text { ship between attitudes towards COVID-19 } \\
\text { vaccine, and conspiracy theories (r: } 0 \cdot 214)\end{array}$ & Very Low \\
\hline
\end{tabular}

Only one study mentioned autism. ${ }^{60}$ In the study by Melton and colleagues, 13 Reddit communities were examined to elucidate COVID-19 related vaccine discussions on social media. They found that the discussions had a more positive than negative sentiment in total. From one of the negative threads, they found comments about autism and misinformation about the COVID-19 vaccine and the technology behind it: "MRNA can do/mean literally anything from protecting you from COVID to sterilization all the way into making you autistic." 60

\section{Discussion}

The 45 included studies about misinformation on social media platforms and the link to vaccine hesitancy suggest that there should be great concern about the association between the two. We identified three overall categories of misinformation, namely medical misinformation, conspiracies, and distrust in vaccine development, although the three are connected and sometimes overlapping, as distrust in the vaccine development might be founded in conspiratorial beliefs about hidden power structures and corrupt elites. The included studies were predominantly from Europe and the US, and there is, therefore, a lack of information from especially African and South American countries. Twitter was the most studied platform, with Facebook and YouTube in second and third place. 
22

Fear of side effects is a major concern when it comes to vaccine-hesitancy, and as our review shows this concern can easily turn into medical misinformation and exaggerations of side effects. Knowing that the side effects of the vaccines are one of the major concerns, medical misinformation has the potential to do a lot of harm. When we examined types of reported misinformation, we also found that a lot of misinformation is grounded in conspiracy theories. For example, we found three studies from the US that mentioned the fear of racist motives by official health authorities as a reason for vaccine hesitancy. ${ }^{42,49,65}$ Some of this fear has historical roots in the US, as one of these studies, ${ }^{65}$ for instance, brought up The Tuskegee Syphilis Study. This was a clinical study (1932-1972) where the US Public Health Service used African Americans to observe untreated syphilis and therefore denied them treatment. ${ }^{67}$ This exemplifies that lack of trust in public health institutions might have deep historical roots in some countries and cultures. Other issues to be aware of are religious concerns and vaccine hesitancy. We found several studies that reported on misinformation about the content and development of the vaccines and used very explicit language (e.g. 'pigs' and 'cells from aborted children'). Such wording can cause worry in some religious communities.

The 19 identified studies in table 3 interpreted results as associations between social media use, misinformation, and vaccine hesitancy. According to the JBI and GRADE evaluations, there is a need for more robust designs to become more certain regarding the actual effect of social media misinformation on vaccine hesitancy. Only one study, an intervention study regarding the impact of addressing misinformation on Twitter users, was assessed to have a low risk of bias and moderate quality of evidence. ${ }^{35}$ In addition, four studies reported significance levels of associations, but the effect size was not reported. ${ }^{28,29,32,58}$ Other studies in this review showed that social media platforms did not necessarily spread misinformation to a great extent, perhaps reflecting that the effort made by some of the social media platforms to halt misinformation has worked. Chan and colleagues ${ }^{44}$ examined 48 COVID-19 vaccine-related videos on YouTube during December 2020 and only found two videos (4.2\%) that made non-factual claims. Hernandez-Garcia and colleagues ${ }^{52}$ also looked at YouTube videos during February 2021 and found that only two out of 110 videos contained COVID-19 vaccine hoaxes or conspiracy theories. Pascual-Ferra and colleagues ${ }^{61}$ examined social media data from Facebook, Instagram, Reddit, and YouTube and did not find evidence of the dominance of misinformation. However, what is being spread and discussed in closed groups is another question that needs to be examined further. Another valid approach would also be to look at the comment sections. Even though anti-vaccine 
content has been prevented to surface in searches, it does not prevent people from commenting on their beliefs or posting other types of information in the comment section. It is not without controversy to de-platform people ${ }^{68}$, and might even do harm, as these people might be seen as someone speaking against the establishment which is in essence some of the core beliefs of some conspiracy theorists.

Surprisingly, there was a dearth of studies examining misinformation about autism and COVID-19 vaccines. Considering the history of misinformation about vaccines and autism during the past two decades, more research should focus on this topic. Future research should also aim at examining social media platforms such as TikTok, which is a very popular platform worldwide, and often used by younger people than for instance the average Twitter user. ${ }^{69}$ Our review shows that more studies are needed from Global South nations.

When addressing vaccine hesitancy, one should be careful before labeling all vaccine-hesitant people as anti-vaxxers or as misinformed. Primary concerns from people who say that they are vaccine-hesitant are the safety of the vaccines and the rapid pace of development. ${ }^{7}$ However, to be hesitant and skeptical does not mean that these people are not willing to take the vaccines, but rather that they have some concerns that should be addressed adequately to convince them of the safety and efficacy of the vaccines. To understand a complex issue such as vaccine hesitancy, knowledge about sociodemographic conditions and cultural awareness is key. In addition, countries with a more un-democratic regime will suffer from a lack of trust in official authorities which may damage an official vaccine campaign. On the other hand, such countries can use authoritarian measures to reach a high vaccination rate. These are issues that need to be addressed and analysed in future studies of vaccine hesitancy.

\section{Limitations}

We did not include grey literature or preprints in our review. The rapid pace at which the pandemic is moving makes preprint research especially relevant. Therefore, including preprints would have been a valid approach. Neither did we contact researchers with potential projects on the subject matter. Further, the databases searched were selected because of the topic at hand. There will always be a chance that other, more specified or general databases would capture other studies. 
24

A limitation of the evidence included in this review is that in our assessment, all but one got a low score in the assessment of risk of bias. Furthermore, there is a poor correlation between self-reported social media use and actual use. ${ }^{70} \mathrm{~A}$ high proportion of these studies harvested data from Twitter because Twitter has opened up access for researchers to extract data from its platform, making it more accessible compared to other social media platforms. The Twitter sample may not be representative of a random sample of the population, as its users tend to range from the age of 25 to 34 years, and are

predominantly from the US. ${ }^{71,72}$ Furthermore, we did not assess the potential presence of social media bots (automated accounts) spreading wrong information in these studies. Neither did we discuss how social media algorithms partake in creating echo chambers. ${ }^{73}$ These are well-known challenges when researching data gathered from social media. ${ }^{74}$

\section{Conclusion}

Many studies showed that there is a link between misinformation on social media and COVID-19 vaccine hesitancy. However, there is a need to examine this with a more robust experimental design to assess this effect. There are many types of misinformation that are spread on social media platforms, and to prevent these myths from taking hold health authorities should openly address and discuss these false claims with both cultural and religious awareness in mind. Although some of the major tech companies have taken steps to prevent misinformation, more action is needed to stop this infodemic. Misinformation about COVID-19 vaccines is still thriving on social media platforms. This undertaking must, however, represent a balance between people's right to speak their minds and strategies to counter the spread of misinformation.

\section{Contributors}

IS, EG, and ANH conceived the idea for this study. Data search was carried out by KEJ. IS and ANH screened titles and abstracts. IS and EG full-text screened the articles. IS did the data extraction, with EG's verification. DQ assessed risk of bias and RW evaluated the quality of evidence. IS took the lead in writing the manuscript. All authors discussed, revised, and contributed to the final manuscript. All authors have read and agreed to the final published version.

\section{Declaration of Interest}

We declare no competing interests. 


\section{Data sharing}

Data will be made available immediately following publication with no end date.

\section{Acknowledgments}

We would like to thank senior librarian Kjell Erik Johnsen for his help and input when doing systematic searches. 


\section{References:}

1 World Health Organization. Ten Threats to Global Health in 2019. 202I. [cited Oct 24, 202I]. Available from https://www.who.int/news-room/spotlight/ten-threats-to-global-health-in-2019

2 Our World in Data. Coronavirus (COVID-19) Vaccinations. England and Wales: Global Change Data Lab. 202I [Updated Dec 3, 2021; cited Dec 3, 202I]. Available from https://ourworldindata.org/covid-vaccinations

3 The New York Times. Tracking Coronavirus Around the World. [internet]. 202I. [Upated on Dec I, 202I; cited on December 3, 202I]. Available from https://www.nytimes.com/interactive/202I/world/covid-vaccinations-tracker.html

$4 \quad$ World Health Organization. Update on Omicron. 2021. [cited Dec 3, 202I]. Available from

https://www.who.int/news/item/28-II-202I-update-on-omicron

5 Machingaidze, S., Wiysonge, C.S. Understanding COVID-19 vaccine hesitancy. Nat Med 2021; 27, I338-1339. https://doi.org/I0.1038/s4I59|-02I-01459-7

6 Gabarron E, Oyeyemi SO, Wynn R. COVID-19-related misinformation on social media: a systematic review. Bull World Health Organ 202I; 99(6):455-463A. doi: 10.247I/BLT.20.276782.

$7 \quad$ World Health Organization. Infodemic. 202I. [cited 202I October 21]. Available from

https://www.who.int/health-topics/infodemic \#tab=tab_ I

8 Tsao SF, Chen H, Tisseverasinghe T, Yang Y, Li L, Butt ZA. What social media told us in the time of COVID-19:

a scoping review. Lancet Digit Health. 2021;3(3):el 75-el 94. doi:I 0. I0I6/S2589-7500(20)30315-0

$9 \quad$ Lord C, Brugha TS, Charman T, et al. Autism spectrum disorder. Nat Rev Dis Primers 2020; 6(I):5. doi:

10.1038/s4I572-019-0I38-4. PMID: 31949|63.

10 Dubé E, Laberge C, Guay M, Bramadat P, Roy R, Bettinger J. Vaccine hesitancy. Human Hum Vaccin Immunother

2013; 9:8, I763-I773, DOI: 10.416I/hv.24657

II Motta M, Stecula D. Quantifying the effect of Wakefield et al. (1998) on skepticism about MMR vaccine safety in the U.S. PLoS ONE 202I; I 6(8): e0256395. https://doi.org/I0.137I/journal.pone.0256395

12 Oyeyemi SO, Gabarron E, Wynn R. Ebola, Twitter, and misinformation: a dangerous combination? BMJ 20I4; 349:g6178. doi: $10.1136 / \mathrm{bmj} . g 6178$

13 Wilson SL, Wiysonge C. Social media and vaccine hesitancy. BMJ Glob Health 2020; Oct;5(10):e004206. doi:

10.1 I36/bmjgh-2020-004206. Epub 2020 Oct 23. PMID: 33097547; PMCID: PMC7590343.

14 World Health Organization. Immunizing the public against misinformation. [internet]. 202I. [cited Oct 2I, 202I].

Available from https:/www.who.int/news-room/feature-stories/detail/immunizing-the-public-against-misinformation

I5 Garritty C, Gartlehner G, Kamel C, King VJ, Nussbaumer-Streit B, Stevens A, Hamel C, Affengruber L. Cochrane Rapid Reviews. Interim Guidance from the Cochrane Rapid Reviews Methods Group. March 2020

16 Page MJ, McKenzie JE, Bossuyt PM, Boutron I, Hoffmann TC, Mulrow CD, et al. The PRISMA 2020 statement: an updated guideline for reporting systematic reviews. BMJ 2020. BMJ 2021;372:n7I. doi: I0.1 I36/bmj.n7|

17 Campbell M, Mckenzie JE, Sowden A, Katikireddi SV, Brennan SE, Ellis S, et al. Synthesis without meta-analysis

(SWiM) in systematic reviews: reporting guideline. BMJ 2020; 16890.

18 Pfizer Inc. Pfizer and BioNTech Conclude Phase 3 Study of COVID-19 Vaccine Candidate, Meeting All Primary

Efficacy Endpoints. 2020. [updated on Nov 18, 2020; cited Jan 18, 2022]. Available from

https://www.pfizer.com/news/press-release/press-release-detail/pfizer-and-biontech-conclude-phase-3-study-covid-19-vac-

cine

19 Braun V, Clarke V. Using thematic analysis in psychology. Qual Res Psychol 2006; 3:2, 77 -

I0I, DOI: 10.1191/1478088706qp063oa

20 Joanna Briggs Institute. JBI Critical Appraisal Checklist for Analytical Cross Sectional Studies 2017. Available from https://jbi.global/sites/default/files/2019-05/JBI_Critical_Appraisal-Checklist_for_Analytical_Cross_Sectional_Studies2017 0.pdf. [Accessed Jan 4, 2022].

2l Schünemann H, Brożek J, Guyatt G, Oxman A, editors. GRADE handbook for grading quality of evidence and strength of recommendations. The GRADE Working Group 2013. Available from guidelinedevelopment.org/handbook. [Accessed November 5, 202I]

22 Alibrahim J, Awad A. COVID-I9 vaccine hesitancy among the public in Kuwait: A cross-sectional survey. Int J Environ $2021 ; 18(16)$.

23 Allington D, McAndrew S, Moxham-Hall V, Duffy B. Coronavirus conspiracy suspicions, general vaccine attitudes, trust and coronavirus information source as predictors of vaccine hesitancy among UK residents during the COVID-19 pandemic. Psychol Med. $2021: 1-12$.

24 Aloweidi A, Bsisu I, Suleiman A, Abu-Halaweh S, Almustafa M, Aqel M, et al. Hesitancy towards covid-19 vaccines: An analytical cross-sectional study. Int J Environ 202।;18(I0). 
25 Bhagianadh D, Arora K. COVID-19 Vaccine Hesitancy Among Community-Dwelling Older Adults: The Role of Information Sources. J Appl Gerontol 202 I; 4 I (I):4-I I. doi: 10.1 I 77/073346482 I I 037507.

26 Brodziak A, Sigorski D, Osmola M, et al. Attitudes of Patients with Cancer towards Vaccinations-Results of Online Survey with Special Focus on the Vaccination against COVID- I9. Vaccines. 202I; 9(5):4II. https://doi.org// 0.3390/vaccines90504II

27 Chadwick A, Kaiser J, Vaccari C, Freeman D, Lambe S, Loe BS, et al. Online Social Endorsement and Covid-19 Vaccine Hesitancy in the United Kingdom. Social Media and Society 2021;7(2).

28 Ebrahimi OV, Johnson MS, Ebling S, Amundsen OM, Halsoy O, Hoffart A, et al. Risk, Trust, and Flawed Assumptions: Vaccine Hesitancy During the COVID-19 Pandemic. Public Health Front. 2021;9: 7002I 3.

29 Kanyike AM, Olum R, Kajjimu J, Ojilong D, Akech GM, Nassozi DR, et al. Acceptance of the coronavirus disease2019 vaccine among medical students in Uganda. Trop Med Health. 2021;49(I).

30 Karabela ȘN, Coșkun F, Hoșgör H. Investigation of the relationships between perceived causes of COVID-19, attitudes towards vaccine and level of trust in information sources from the perspective of Infodemic: the case of Turkey. BMC Public Health 2021; 2 I(I): I 195. doi: 10.1 I86/s I 2889-02 I-I I 262-I.

3I Park S, Massey PM, Stimpson JP. Primary Source of Information About COVID-19 as a Determinant of Perception of COVID-19 Severity and Vaccine Uptake: Source of Information and COVID-19. J Gen Intern Med 202I; I-8.

32 Petravić L, Arh R, Gabrovec T, et al. Factors Affecting Attitudes towards COVID-19 Vaccination: An Online Survey in Slovenia. Vaccines 202I; 9(3):247. doi: 10.3390/vaccines9030247.

33 Sallam M, Dababseh D, Eid H, Hasan H, Taim D, Al-Mahzoum K, et al. Low COVID-19 Vaccine Acceptance Is Correlated with Conspiracy Beliefs among University Students in Jordan. Int J Environ Res Public Health 2021; I 8(5).

34 Sallam M, Dababseh D, Eid H, et al. High Rates of COVID-19 Vaccine Hesitancy and Its Association with Conspiracy Beliefs: A Study in Jordan and Kuwait among Other Arab Countries. Vaccines (Basel). 2021;9(I):42. Published 202 I Jan I2. doi:I0.3390/vaccines 9010042

35 Sharevski F, Gover D. Two Truths and a Lie: Exploring Soft Moderation of COVID-19 Misinformation with Amazon Alexa. In The 16th International Conference on Availability, Reliability and Security (ARES 202I). Association for Computing Machinery, New York, NY, USA, Article 79, I-9. DOI:https://doi.org/ I 0.I I 45/346548I.34700I7

36 Zhang KC, Fang Y, Cao H, Chen H, Hu T, Chen Y, et al. Behavioral intention to receive a COVID- 19 vaccination among Chinese factory workers: Cross-sectional online survey. JMIR 2021;23(3).

37 Zhang KC, Fang Y, Cao H, Chen H, Hu T, Chen YQ, et al. Parental acceptability of COVID-19 vaccination for children under the age of 18 years: Cross-sectional online survey. JMIR Pediatr Parent 2020;3(2).

38 Costantino C, Gori D, Alba D, Cimino L, Conforto A, Tomasello F, et al. Willingness to receive covid- 19 vaccination in costumers accessing community pharmacies in the province of Palermo, Italy. EuroMediterranean Biomed J 2021;16(19):80-4.

39 El-Far Cardo A, Kraus T, Kaifie A. Factors That Shape People's Attitudes towards the COVID-I9 Pandemic in Germany-The Influence of MEDIA, Politics and Personal Characteristics. Int J Environ Res Public Health 202I; I 8(I5).

40 Jennings W, Stoker G, Bunting H, Valgarðsson VO, Gaskell J, Devine D, et al. Lack of Trust, Conspiracy Beliefs, and Social Media Use Predict COVID- 19 Vaccine Hesitancy. Vaccines (Basel). 2021;9(6).

$4 I \quad K n i g h t s ~ F$, Carter J, Deal A, Crawshaw AF, Hayward SE, Jones L, et al. Impact of COVID-19 on migrants' access to primary care and implications for vaccine roll-out: A national qualitative study. British Journal of General Practice. 202 I;7 I (709):E583-E95.

42 Berry SD, Johnson KS, Myles L, Herndon L, Montoya A, Fashaw S, et al. Lessons learned from frontline skilled nursing facility staff regarding COVID-19 vaccine hesitancy. J Am Geriatr Soc 2021; 69(5): I I40-6.

43 Choudhary K, Garapati P, Prasad SR, Murti K, Singh S, Kumar N. COVID-I 9 vaccine hesitancy assessment among the educated Indian population: An online survey. Int J Pharm Res. 202 I; I 3(3):I358-63.

44 Chan C, Sounderajah V, Daniels E, Acharya A, Clarke J, Yalamanchili S, et al. The reliability and quality of youtube videos as a source of public health information regarding COVID-19 vaccination: Cross-sectional study. JMIR Public Health Surveill 2021;7(7).

45 Alliheibi FM, Omar A, Al-Horais N. Opinion Mining of Saudi Responses to COVID-19 Vaccines on Twitter: A Computational Linguistic Approach. Int J Adv Comput Sci Appl 2021;1 2(6):72-8.

46 Baines A, Ittefaq M, Abwao M. \#Scamdemic, \#Plandemic, or \#Scaredemic: What Parler Social Media Platform Tells Us about COVID-19 Vaccine. Vaccines (Basel). 202I; 9(5): 42I. https://doi.org/I0.3390/vaccines905042I.

47 Basch CH, Meleo-Erwin Z, Fera J, Jaime C, Basch CE. A global pandemic in the time of viral memes: COVID-19 vaccine misinformation and disinformation on TikTok. Hum Vaccin Immunother. 202I; I7(8):2373-2377. doi:

10.1080/21645515.2021.1894896. 
48 Boucher JC, Cornelson K, Benham JL, Fullerton MM, Tang T, Constantinescu C, et al. Analyzing Social Media to Explore the Attitudes and Behaviors Following the Announcement of Successful COVID-19 Vaccine Trials: Infodemiology Study. JMIR Infodemiology 202I; I (I):e28800.

49 Criss S, Nguyen TT, Norton S, et al. Advocacy, Hesitancy, and Equity: Exploring U.S. Race-Related Discussions of the COVID-I 9 Vaccine on Twitter. Int J Environ Res Public Health. 202 I; I 8(I I):5693. doi: 10.3390/ijerph I8I I 5693.

50 Doyno CR, Fitzgerald JM, White CM, Sobieraj DM, Zacchera M. (in press). Innovative partnership in Connecticut to expand health professional eligibility to administer COVID-19 vaccines. Journal of the American Pharmacists Association. J Am Pharm Assoc 202I.

5I Guntuku SC, Buttenheim AM, Sherman G, Merchant RM. Twitter discourse reveals geographical and temporal variation in concerns about COVID-19 vaccines in the United States. Vaccine 2021; 39(30):4034-8.

52 Hernández-García I, Gascón-Giménez I, Gascón-Giménez A, Giménez-Júlvez T. Information in Spanish on YouTube about Covid-19 vaccines. Hum Vaccin Immunother 202I:I-6.

53 Herrera-Peco I, Jiménez-Gómez B, Romero Magdalena CS, Deudero JJ, García-Puente M, Benítez De Gracia E, et al. Antivaccine movement and covid-19 negationism: A content analysis of spanish-written messages on twitter. Vaccines. $2021 ; 9(6)$.

54 Herrera-Peco I, Ruiz Núñez C, Jiménez-Gómez B, Romero-Magdalena CS, Benítez De Gracia E. COVID-19 and vaccination: analysis of public institution's role in information spread through Twitter. Revista espanola de salud publica $2021 ; 95$.

55 Hughes B, Miller-Idriss C, Piltch-Loeb R, Goldberg B, White K, Criezis M, et al. Development of a codebook of online anti-vaccination rhetoric to manage covid-19 vaccine misinformation. Int J Environ Res 2021; I 8(I4).

56 Islam MS, Kamal AM, Kabir A, Southern DL, Khan SH, Hasan SMM, et al. COVID-I9 vaccine rumors and conspiracy theories: The need for cognitive inoculation against misinformation to improve vaccine adherence. PLoS One 2021; 16(5):e025I605.

57 Kwok SWH, Vadde SK, Wang G. Tweet Topics and Sentiments Relating to COVID-19 Vaccination Among Australian Twitter Users: Machine Learning Analysis. J Med Internet Res 2021;23(5):e26953.

58 Larrondo-Ureta A, Fernández SP, Morales-I-gras J. Disinformation, vaccines, and covid-19. Analysis of the infodemic and the digital conversation on twitter. Revista Latina de Comunicacion Social 202 I; 79: I-I8.

59 Liu S, Liu J. Understanding Behavioral Intentions Toward COVID-19 Vaccines: Theory-Based Content Analysis of Tweets. J Med Internet Res 2021;23(5):e28I 18

doi: $10.2196 / 28118$

60 Melton CA, Olusanya OA, Ammar N, Shaban-Nejad A. Public sentiment analysis and topic modeling regarding COVID-19 vaccines on the Reddit social media platform: A call to action for strengthening vaccine confidence. J Infect Public Health 202I; I 4(I0):I505-I5I2. doi:I0. I0I6/j.jiph.202I.08.0I0

61 Pascual-Ferrá P, Alperstein N, Barnett DJ. A Multi-platform Approach to Monitoring Negative Dominance for COVID- 19 Vaccine-Related Information Online [published online ahead of print, 202I May 3]. Disaster Med Public Health Prep. 2021;I-24. doi:10.1017/dmp.202I.136

62 Rotolo SM, Jain S, Dhaon S, Dokhanchi JK, Kalata E, Shah T, et al. (in press). A coordinated strategy to develop and distribute infographics addressing COVID-19 vaccine hesitancy and misinformation. J Am Pharm Assoc (2003) 2021.

63 Savolainen R. Assessing the credibility of COVID-19 vaccine mis/disinformation in online discussion. Journal of Information Science 2021. doi:10.1 I77/01655515211040653

64 Sobkowicz P, Sobkowicz A. Agent Based Model of Anti-Vaccination Movements: Simulations and Comparison with Empirical Data. Vaccines (Basel) 2021;9(8):809. Published 2021 Jul 21. doi:I0.3390/vaccines9080809

65 Thelwall M, Kousha K, Thelwall S. Covid-19 vaccine hesitancy on English-language Twitter. Profesional de la Informacion 2021;30(2). e3002I2. https://doi.org/I0.3145/epi.202I.mar.12

66 Wawrzuta D, Jaworski M, Gotlib J, Panczyk M. What arguments against COVID-19 vaccines run on facebook in poland: Content analysis of comments. Vaccines 2021;9(5).

67 Centers for Disease Control and Prevention (CDC). The U.S Health Service Syphilis Study at Tuskegee. The Tuskegee Timeline. [internet]. National Center for HIV, Viral Hepatitis, STD, and TB Prevention, Centers for Disease Control and Prevention. 202I. [Updated April 22, 2021 ; cited Nov 4, 202I]. Available at

https://www.cdc.gov/tuskegee/timeline.htm

68 Burki T. The online anti-vaccine movement in the age of COVID-19. Lancet Digit Health. 2020;2(I0):e504-e505. doi:10.1016/S2589-7500(20)30227-2

69 Statista. Most popular social networks worldwide as of October 2021, ranked by number of active users (in millions). [internet]. Statista Research Department. 202I. [Updated November 14, 202 I; cited Jan 3, 2022]. Available from https://www.statista.com/statistics/2720I4/global-social-networks-ranked-by-number-of-users/ 
70 Parry, D. A., Davidson, B. I., Sewall, C. J., Fisher, J. T., Mieczkowski, H., \& Quintana, D. S. A systematic review and meta-analysis of discrepancies between logged and self-reported digital media use. Nat Hum Behav 202I; I-I3.

71 Statista. Distribution of Twitter users worldwide as of April 202I, by age group. [internet]. Statista Research Department. 2021. [Updated Sept 7, 2021 ; cited Jan 3, 2022]. Available from_ https://www.statista.com/statistics/283। I 9/agedistribution-of-global-twitter-users/

72 Tankovska H. Leading Countries based on number of Twitter users as of October 202I (in millions). Statista. [Updated Nov 19, 2021; Accessed Jan 4, 2022]. https://www.statista.com/statistics/242606/number-of-active-twitter-usersin-selected-countries/

73 Cinelli M, Morales GD, Galeazzi A, Quattrociocchi W, Starnini M. The echo chamber effect on social media. Proceedings of the National Academy of Sciences. 202I Mar 2; I I 8(9).

74 Orabi M, Mouheb D, Al Aghbari Z, Kamel I. Detection of Bots in Social Media: A Systematic Review. Inf Process Manag 2020;57(4): 102250. 